\title{
Outcome of Laparoscopic Deroofing of Renal Cyst- Our Experience
}

\author{
Md. Nazmul Haque *1, Md. Muazzam Hossan ${ }^{2}$, Md. Mofiz Uddin ${ }^{3}$
}

\begin{abstract}
Introduction: To evaluate the outcome of laparoscopic deroofing of renal cyst for the management of symptomatic renal cyst. Materials \& Methods: This study was performed on 32 patients having symptomatic renal cyst at the Department of Urology, Shahid Sheik Abu Naser Specialized Hospital, Khulna and two other private hospitals in Khulna from January 2015 to December 2017. Patients having cyst size less than 5cm, previous abdominal surgery and sepsis were excluded from this study. Transperitoneal approach was adopted in all cases. All patients were diagnosed by ultrasonography and computed tomography to determine the Bosniak classification of the cyst. Pain and cyst recurrence were assessed during the follow-up. Results: Thirty-two patients (mean age $=52.9 \pm 12$ years) with large renal cyst (mean size $=35 \pm 13$ ) were included. All procedures were completed successfully, with no major intraoperative complications. The mean (range) operative duration was 56 (35-125) min, affected by the site and number of cysts unroofed. All patients were symptom-free except one, who had a recurrent large cyst, anteriorly located, and who underwent open cyst deroofing. Conclusion: Laparoscopic deroofing of symptomatic renal cysts should be the standard of care, especially after failed percutaneous aspiration, with reduced postoperative pain, short hospital stay and cost effective. It is feasible with conventional laparoscopic instruments and gives a better cosmetic outcome.
\end{abstract}

Keywords: Renal cyst, Laparoscopic decortication.

Number of Tables: 01; Number of References: 10; Number of Correspondences: 03

*1. Corresponding Author: Dr. Md. Nazmul Haque

Assistant Professor

Department of Urology

Shahid Sheikh Abu Naser Specialized Hospital Khulna, Bangladesh.

\section{Md. Muazzam Hossan}

Assistant Professor

Department of Urology

Shahid Sheikh Abu Naser Specialized Hospital Khulna, Bangladesh.

3. Dr. Md. Mofiz Uddin

Assisstant Registrar

Department of Urology

Shahid Sheikh Abu Naser Specialized Hospital

Khulna, Bangladesh.

\section{Introduction}

Renal cysts are common and can represent a manifestation of an inherited or acquired disorder. Simple cysts are rare in childhood, but increase in frequency during adulthood ${ }^{1}$. The increasing incorporation of imaging into urological practice has produced a corresponding increase in the detection of renal cysts ${ }^{2}$.

The indications for surgical intervention for renal cysts are pain, infection, hypertension, haemorrhage, collecting-system obstruction, or the risk of malignancy. The treatment options for symptomatic cysts include aspiration with or without instillation of sclerosing agents, percutaneous resection, and open or laparoscopic deroofing ${ }^{3}$.

Laparoscopic management has become the standard of care because it is minimally invasive, cosmetically acceptable and has a high success rate in terms of cyst recurrence ${ }^{4}$. We present our experience in treating symptomatic renal cysts by laparoscopic deroofing, considering the morbidity and clinical outcome.

\section{Materials and Methods}

Between January 2015 and December 2017, 32 patients (22 males and 10 females) underwent laparoscopic renal cyst deroofing, with 5 of them having recurrent cysts after percutaneous aspiration. The mean (range) age of the patients was 46 (17-65) years. Eleven patients had right renal cysts, 12 had left renal cysts. The mean (range) size of the cysts was $10.8(8-16) \mathrm{cm}$. Transperitoneal approach was used in all patients. The patients' demographic data are summarised in Table I.

The main presenting symptoms were renal pain in all patients, urinary tract obstruction in two, with microscopic haematuria due to lower polar cysts obstructing the upper ureter. After detecting the cysts with abdominal ultrasonography, CT with a renal-mass protocol was used and showed Bosniak type I or II cysts in all cases. Small unsymptomatic simple cysts $(<5 \mathrm{~cm})$ and renal cysts of higher grade 
(type IIF, III and IV Bosniak classification) were excluded. Urine analysis with culture and sensitivity was assessed in all patients, and urine cytology was assessed in those with haematuria.

The timeline of the procedures conforms to the development of our laparoscopic experience. All patients were operated via transperitoneal approach. After induction of anesthesia, patients were in flank position with no flexion of the operating table. The procedure was performed through 3 ports, a $10 \mathrm{~mm}$ camera trocar inserted 2 fingerbreadth lateral and superior to the umbilicus, and 2 additional $5 \mathrm{~mm}$ working ports inserted a handbreadth superior and inferior to the camera port. A fourth port was added in some patients, usually those with anterior upper-pole cysts. After reflection of the colon, the cyst was identified and it appeared in most cases as a blue dome, which was then dissected and its edge was delivered. The cyst contents were aspirated by a percutaneous needle under laparoscopic guidance, and the aspirate was sent for cytological analysis. The roof of the cyst was then excised with endoscissors and submitted together with several 'bites' from the floor of the cyst for a histopathological examination. The cyst edge was sealed by electrocautery, and the perirenal fat was placed over the base of the cyst. Finally, a tube drain was left and the wounds were closed.

\section{Results}

All procedures were completed successfully, with no major intraoperative complications and no blood transfusions needed. The mean (range) operative duration was 56 (35-125) min. There was a gonadal vessel injury in one patient that was secured. Oral feeding started $24 \mathrm{~h}$ after surgery but was delayed to $48 \mathrm{~h}$ in two patients (8.7\%) due to a mild ileus that resolved spontaneously. There was a low-grade fever in three patients (13.0\%). There were no urinary leakages or retroperitoneal collections. For postoperative analgesia we used ketorolac, with a mean (range) dose of $25.2(0-60) \mathrm{mg}$ daily. The tube drain was removed 1-2 days after surgery. The hospital stay was $2-5$ days and the return to normal activity was at $1-3$ weeks.

Percutaneous aspiration under laparoscopic guidance showed a clear fluid, with negative cytology in all cases. No malignancy was detected on histopathological examination of the cyst walls. The patients were followed up for 6-12 months, with a clinical assessment for pain, and by abdominal ultrasonography. All patients were symptom-free, with no urinary tract obstruction. Early in our laparoscopic experience there was a recurrence in one patient who had an anterior upper-pole cyst of $7 \mathrm{~cm}$ in diameter, which was managed by open surgical deroofing because of extensive peritoneal adhesions. The various complications are also summarized in Table I.
Table-I: Demographic profile and outcome among cases $(\mathrm{n}=32)$.

\begin{tabular}{lll}
\hline Parameters & Variables & $\mathbf{n}(\%)$ \\
\hline Gender & Male & $22(68.75)$ \\
& Female & $10(31.25)$ \\
Side of Cyst & Right & $18(56.25)$ \\
& Left & $14(43.75)$ \\
Presentation & Renal pain & $32(100)$ \\
& Obstruction & $2(6.250)$ \\
Mode of Surgery & Transperitoneal & $32(100)$ \\
(Laparoscopy) & & \\
Complications, & II, Gonadal & $1(3.125)$ \\
by grade & vessel injury & \\
& I, Fever & $3(9.375)$ \\
& I, Ileus & $2(6.250)$ \\
& II, Perinephric & $1(3.125)$ \\
& haematoma & \\
\hline
\end{tabular}

\section{Discussion}

Laparoscopic cyst deroofing is an effective and durable treatment for symptomatic renal cysts, as assessed over a long-term follow-up. Its minimal invasiveness and greater success rate favour it over other treatments 5 .

The percutaneous aspiration of simple renal cysts is an easy and safe procedure, and can be used to ascertain if cyst decompression results in the resolution of pain, and if not, then to justify further more invasive treatment; however, it is associated with high rate of recurrence. The percutaneous instillation of sclerosing agents into simple renal cysts is associated with success rates of $75-97 \%$, with a complication rate of $1.3-20 \%{ }^{6}$. In the present study we included 5 patients with a recurrence after percutaneous aspiration. The percutaneous resection of renal cysts also has been advocated. The long-term results showed a 30\% recurrence rate and $20 \%$ residual cysts ${ }^{7}$.

Some authors recommended that retrograde pyelography should be used just before the start of the laparoscopic procedure, especially in those with parapelvic and parenchymal cysts, to assess cyst communication with the collecting system, and possibly an injection with methylene blue via a ureteric catheter for a final check at the end of the maneuver. However, we think that this is not mandatory and we did not use this in our series where there were no significant complications ${ }^{8}$.

The retroperitoneal approach reduces the risk of hypercarbia, hypothermia, postoperative ileus, inadvertent intra-abdominal organ injury and hernia formation, compared to the transperitoneal approach, which was not adopted in this study'.

Cysts can recur, possibly due to incomplete handling or 
incomplete excision of the cyst wall. To decrease the possibility of recurrence, the renal cyst wall should be completely excised if possible. If it is not possible to excise the cyst wall completely, the perinephric fat should be tucked into the cavity ${ }^{10}$. We report one case of recurrence, which was managed by open surgical deroofing.

For urologists, the retroperitoneal approach is preferable and familiar, hence many surgeons started retroperitoneal approach. But in this study we used transperitoneal approach, as it provides a wide working space, can be applied bilaterally in the same session, and is more cosmetically acceptable.

\section{Conclusion}

In conclusion, the laparoscopic deroofing of symptomatic renal cysts should be the standard of care, especially after failed percutaneous aspiration or decortication. It is feasible with conventional laparoscopic instruments and has a better cosmetic outcome.

Laparoscopicderoofing of renal cyst is a safe and effective approach to treat such disease. It has less morbidity, less hospital stay and better patient compliance. It should be considered as the treatment option of treating renal cysts.

\section{Conflict of Interests: None.}

\section{Acknowledgement}

We are highly grateful to Director, Department of Anesthesiology and operation theatre stuff of Shahid Sheikh Abu Naser Specialized Hospital, Khulna for their cordial help.

\section{References}

1. Hanash K.A., Al-Othman K., Mokhtar A., Al-Ghamdi A., Aslam M. Laparoscopic ablation of giant renal cyst. J Endourol. 2003; 17: 781-784.

https://doi.org/10.1089/089277903770802371

PMid: 14642043

2. McHugh K., Stringer D.A., Hebert D., Babiak C.A. Simple renal cysts in children. Diagnosis and follow up with ultrasound. Radiology. 1991; 178: 383-385. https://doi.org/10.1148/radiology.178.2.1987597 PMid:1987597
3. Rane A. Laparoscopic management of symptomatic simple renal cysts. IntUrolNephrol. 2004; 36: 5-9.

https://doi.org/10.1023/B:UROL.0000032669.21924.d1

PMid:15338663

4. Hulbert J.C. Laparoscopic management of renal cystic disease. Semin Urol. 1992; 10: 239-241.

5. Atug F., Burgess S.V., Ruiz-Deya G., Mendes-Torres F., Castle E.P., Thomas R. Long-term durability of laparoscopic decortication of symptomatic renal cysts. Urology. 2006; 68: 272-275.

https://doi.org/10.1016/j.urology.2006.03.009

PMid:16904433

6. Pearle M.S., Traxer O., Cadeddu J.A. Renal cystic disease: laparoscopic management. UrolClin North Am. 2000; 27: 661-673.

https://doi.org/10.1016/S0094-0143(05)70116-6

7. Kang Y., Noble C., Gupta M. Percutaneous resection of renal cysts. J Endourol. 2001; 15: 735-738.

https://doi.org/10.1089/08927790152596343

PMid:11697407

8. Roberts W.W., Bluebond-Langner R., Boyle K.E., Jarrett T.W., Kavoussi L.R. Laparoscopic ablation of symptomatic parenchymal and peripelvic renal cysts. Urology. 2001; 58: 156-159.

https://doi.org/10.1016/S0090-4295(01)01145-1

9. Ferzli G., Raboy A., Kleinerman D., Albert P. Extraperitoneal endoscopic pelvic lymph node dissection vs. laparoscopic lymph node dissection in the staging of prostatic and bladder carcinoma. J Laparoendose Surg. 1992; 2: 219-222.

https://doi.org/10.1089/1ps.1992.2.219

PMid:1421539

10. Porpiglia F., Fiori C., Billia M., Renard J., Di Stasio A., Vaccino D. Retroperitoneal decortication of simple renal cysts vs decortication with wadding using perirenal fat tissue: results of a prospective randomized trial. BJU Int. 2009; 103: 1532-1536.

https://doi.org/10.1111/j.1464-410X.2008.08294.x

PMid:19154478 